\title{
Compose signal of time-domain electromagnetic response excited by Pseudo Random Binary Sequence Source
}

\section{Compose signal of TDEM response excited by PRBS}

\author{
Ruo Wang *, Miaoyue Wang, Qingyun Di, \\ Guoqiang Xue, Da Lei \\ Key laboratory of shale gas and engineering geology \\ Institute of Geology and Geophysics, CAS \\ Beijing, 100029 China
}

\author{
Changchun Yin \\ College of geo-exploration science and technology \\ Ji Lin university \\ Changchun 130026
}

\begin{abstract}
Pseudo-Random binary sequence source is introduced to artificial electromagnetic method, it is necessary to use special technique to process the signal in time domain. In order to test the quality of data processing technology, it is indispensable to compose electromagnetic signal of simulated model under pseudo random binary sequence (PRBS) source exciting. In this paper, based on the physical propagated process of the electromagnetic signal, we compose the simulated signal in time domain. Comparing with actual field signal, the composed signal and the field signal is similar to each other, it proved that the composed signal is right, and the high simulated signal can be used in the processing, and can be used to test the effectiveness of the data processing technique.
\end{abstract}

Keywords—pseudo random coded technology; time domain; impulse response; received signal; compose.

\section{INTRODUCTION}

In the past decades, traditional electromagnetic (EM) method which mainly include Audio-frequency magnetotelluric (AMT) method, Control Source Audiofrequency magnetotelluric (CSAMT) method and transient electromagnetic (TEM) method have been used extensively in exploration of energy, mine, water, environmental geology and engineering geology. However, these methods are limited to be used because of their shallow exploration depth and precision, and new methods or techniques need be developed to improve the exploration depth and precision to detect the deeper target.

Recently, Wright ${ }^{[1]}$ mentioned the concept of multichannel TEM (MTEM) and developed the processing technique to detect the gas target. Reference ${ }^{[2]}$ developed wide-domain electromagnetic method to improve the exploration depth and precision through enhancing the transmitter strength and increasing the vertical iteration times.

In all of the new methods, the pseudo random binary sequence (PRBS) has been introduced; these new methods are noticed and studied by some researcher in china ${ }^{[3-6]}$.

Supported by Project on the Development of Key Equipment (ZDYZ2012-1-05) from Chinese Academy of Science and by National Natural Science Foundation of China under the grants 41174111, 41274121.
In the published papers, authors focused more on the relevant identification and suppression noise, and discussed the identification method more than the simulation. However, simulation is not only a essential stage for a new method to know about it but also a base to study the processing and the inversion techniques. Here, we simulate the electromagnetic signal generated by m-PRBS source because the preprocessing technique is based on it.

\section{THEORY}

In this paper, the simulation is to composite an observation signal. At first, an m-PRBS is generated by a series of shift registers, and then the response of the ground is modeled which is integrating the response of the source generated by a m-PRBS code, receiver system and noise, at last a simulated field signal is composited.

The expression of the collected signal can be written as ${ }^{[7]}$ :

$$
v(t)=i(t) * g(t) * r(t)+n(t)
$$

where, the variable $v$ is the signal collected at the receiver, the variable $\mathrm{i}$ is the current transmitted to the ground, the variable $g$ is the ideal impulse response of the ground, the variable $r$ is the band-limit response of the instrument, and the noise is expressed as $n$, all of the variable are the function of time $\mathrm{t}$.

From expression (1), if the ideal impulse response $g(t)$ of the ground, the m-PRBS $i(t)$, the band limit response $r(t)$ and the noise $n(t)$ are obtained, the receiver signal $v(t)$ will be composted.

According to the propagation routine, in the field work, the useful signal is the impulse response of the ground, the distortion signal is the polarization effect around the electrode, the band limited of the receiver, the inductive reactance of the wire, and noise et al., so the signal collected by the receiver is got through the ideal response of ground convoluted by multifilters. Therefore, the composited signal should be considering 
all of the distortion, however, some distortion is relative small or not easy to simulated, therefore, we only consider noise and band- limit response of the instrument which includes the transmitter and the receiver system.

\section{IMPULSE RESPONSE OF GROUND}

In numerical simulation, the response of the ground in frequency domain is obtained first, and then it is transferred to time domain, and the impulse response of ground will be got through the time derivation if the time domain result is step response.

In the paper, the homogeneous half space is used to simulate the electrical field. According to reference 8, only the in-line electrical field can offer useful information, other fields can't give more information, therefore, only the in-line electrical field is used in the paper.

There are many methods can be used to transform data in frequency domain to time domain, such as sine transform or cosine transform, polygon method, and so on, we choose the cosine transform in the paper.

\section{M-PRBS SOURCE}

M-PRBS are generated by shift registers. In simulation, it can be got from sequence polynomial $G(x)$, which is calculated from the primitive polynomial $F(x)$ using long division algorithm, $G(x)=1 / F(x)$, and the primitive polynomial $F(x)$ can be got by table look-up scheme according to the published book ${ }^{[9]}$, the expression of sequence polynomial $G(x)$ is written as:

$$
G(x)=a_{0} \oplus a_{1} x \oplus a_{2} x^{2} \oplus \ldots=\sum_{n=1}^{N} a_{n} x^{n}
$$

where, symbol $\oplus$ is the operator of binary addition, variable $\mathrm{x}$ is the shifting operator, the number of the subscript is the shifting bit, such as, expression $a_{k} x^{2}$ means $a_{k}$ is shifted two bits. The sequence $\left\{a_{n}\right\}=a_{0}, a_{1}, a_{2}, a_{3}, \ldots$ is the sequence of the shifting register, which is the m-sequence code. The letter $\mathrm{N}$ in expression (2) is the number of the code element in one period, and $N=2^{r}-1$, where, $r$ is the rank of the shifting register.

\section{OTHER IMPACT FACTOR}

\section{A. The band-limit effect of instrument}

The instrument of transmitter and receiver is of bandlimited, which can be simulated by a low pass filter. There are some methods to design a filter, and different method could cause different amplitude of the side lobe and different width of the transition band which is the band between the main lobe and the side lobe. The transition band of the ideal filter is narrow enough that the energy could be remained most in the main lobe and leave off less in the side lobe. In this paper, the Hamming window is chosen because $99.963 \%$ energy is remained in the main lobe and less $1 \%$ energy is in the side lobe ${ }^{[7]}$.

\section{B. Adding noise}

We simulate the white noise using Gaussian distribution and simulate the industry noise by using sin function with frequency $50 \mathrm{~Hz}$.

\section{COMPOSITION OF ELECTROMAGNETIC SIGNAL UNDER EXCITING OF THE PSEUDO-RANDOM SOURCE}

When integrating the relevant items discussed above, the ideal receiver signal, the band-limited signal and noisy signal could be obtained respectively. If the source is exiting by mPRBS with 4-rank register as shown in figure 1a, the ideal signal (useful signal shown in Fig.1b) could be got by convoluting the source with the impulse response of the homogeneous half space as shown in fig.1d, and the bandlimited signal is shown in fig.1c. From these figures, we find the signal is rouge after considering land-limit while smooth before.

Fig. $2 \mathrm{a}$ and $2 \mathrm{~b}$ show the signal after adding white noise level $5 \%$ and $10 \%$ respectively to the band-limit signal When the noise level is up to $10 \%$, the distortion is obvious. When adding $50 \mathrm{~Hz}$ industry noise as shown in Fig.2c, the original signal is difficult to be recognized because the amplitude of the industry noise is 4 times to it.
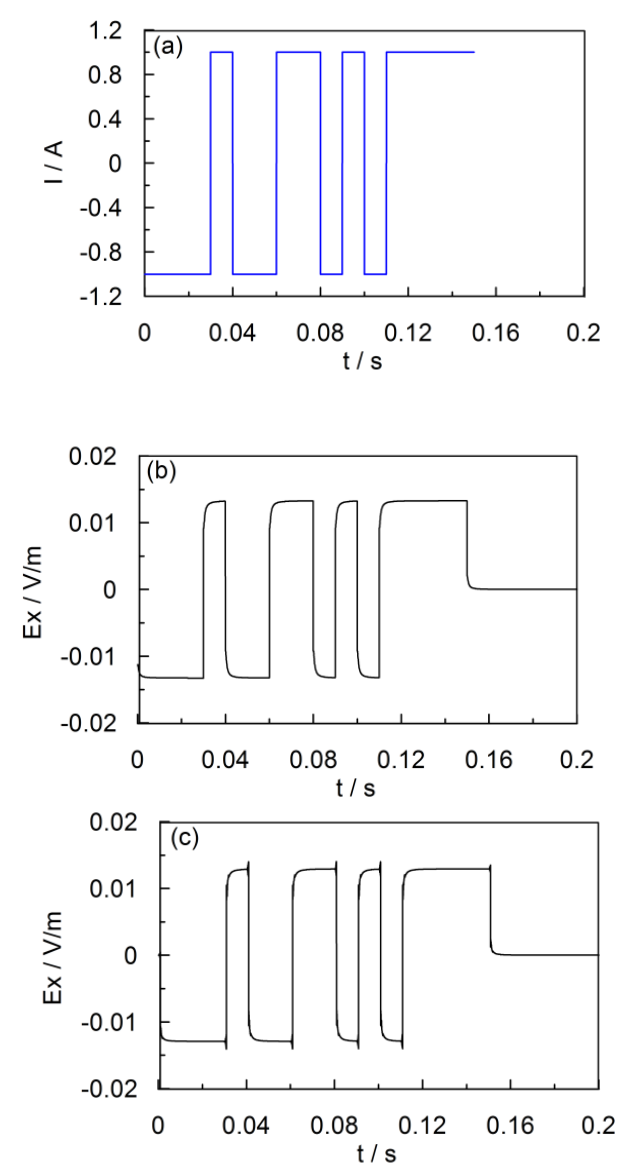
(d)

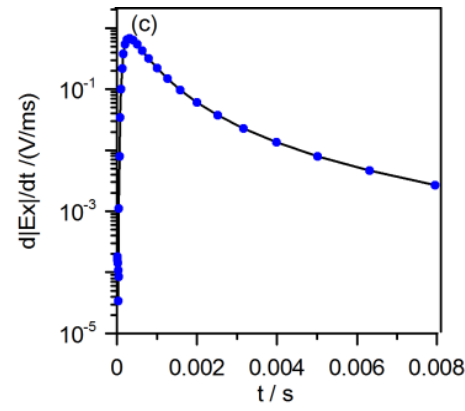

Fig. 1. the signal exciting by 4 rand Pseudo-Random binary sequence $m$ PRBS code source (b) ideal signal (c) the signal considering the band limit (d) the impulse response of homogeneous half space
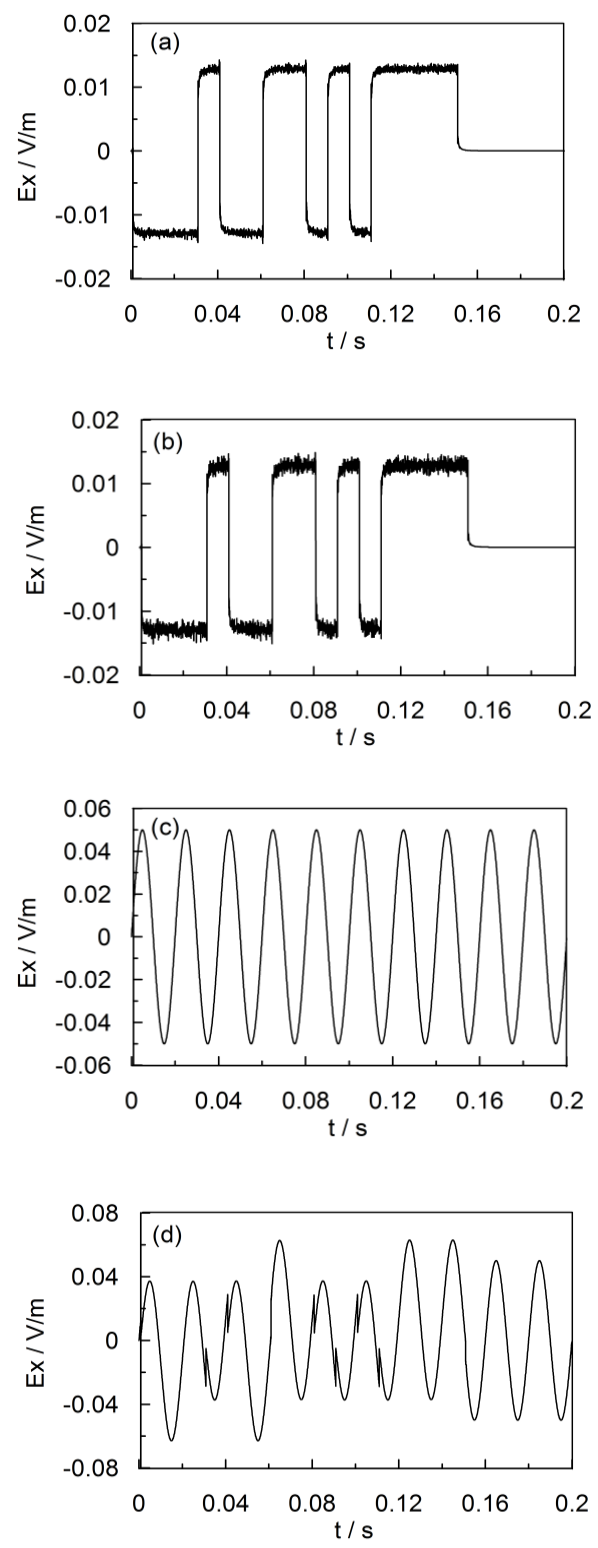

Fig. 2 the signal adding different noise adding 5\% white noise (b) adding $10 \%$ white noise (c) the $50 \mathrm{~Hz}$ industry noise (d) the signal after adding (c) to (b)
In 2005, the group of CAS performed a test using m-PRBS source in Zhang bei, Hebei province, they used a grounded dipole with $300 \mathrm{~m}$ length to inject current with 12-rank mPRBS code to the ground, and received the signal at different offsets. In order to compare the field signal, we simulate the signal using computer, according to the estimated parameter, we assume the ground is homogeneous half space with resistivity $100 \mathrm{Ohm}-\mathrm{m}$, and use 12-rank m-PRBS source similar to the field source as shown in fig. $3 \mathrm{a}$, where only part of the source is shown because the code length is too long. However, the source used in the computer and filed work are not same seriously because the code is different if using different primitive polynomial, the large the rank of register, the more the number of the primitive polynomial is. The parameter of the sample is same as the field work, that is, the code element is $1 / 1024 \mathrm{~s}$, the sample rate is $16 \mathrm{kHz}$, and the period of the source is $4 \mathrm{~s}$. The impulse response simulated at offset $300 \mathrm{~m}$ and $2700 \mathrm{~m}$ are shown in fig. $7 \mathrm{~b}$ and fig. $7 \mathrm{c}$, the response at offset $300 \mathrm{~m}$ is larger than at offset $2700 \mathrm{~m}$, and their form is different, because the signal is up to saturation when the receiver is close to source, otherwise the form is gentle and the time of the peak appear at late time when the receiver is far from the source.

We compose the signal and compare it with the field data. The composed and observed signal of at offset $300 \mathrm{~m}$ is shown in fig. $3 \mathrm{~d}$ and $3 \mathrm{e}$ respectively, where one can find the signal of the composed and observed are similar but not same, there are three reasons, one is the different m-PRBS code, one is the different resistivity of the ground and the simulated, the last is the distortion, which is complex in the field work. However, the similarity of the two kinds of signals proof the composed method is right.

\section{CONCLUSION}

We compose the receiver signal according to the physical propagation procedure of the electromagnetic field exciting by the m-PRBS. The impulse response is calculated from the response in frequency domain and the composed signal is close to the observed signal. Therefore, the composed method in the paper can be used to the data processing to test the reliability and the effective of the processing techniques. 
(a)

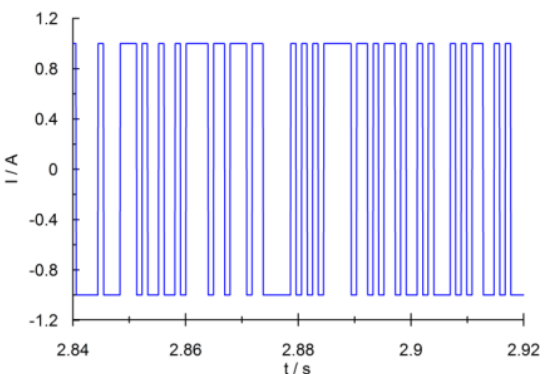

(b)

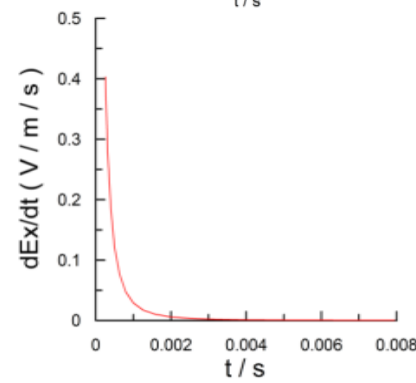

(c)

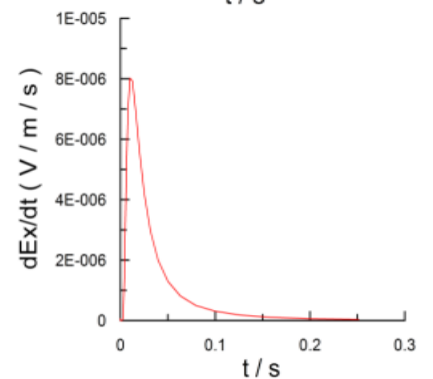

(d)

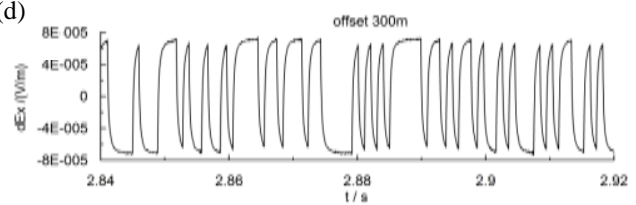

(e)

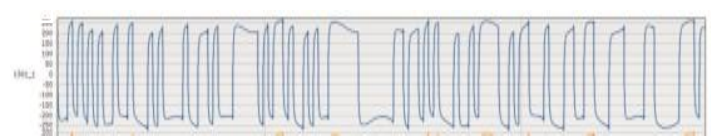

Fig.3. comparison of simulated signal to survey signal (a)source (b) Ex impulse response at offset $300 \mathrm{~m}$ (c) Ex impulse response at offset $2700 \mathrm{~m}$ (d) the simulated signal at offset $300 \mathrm{~m}$ (e) the survey signal at offset $300 \mathrm{~m}$

\section{Acknowledgments}

The paper is financially supported by Project on Development of the Key Equipment (ZDYZ2012-1-05) from Chinese Academy of Science and by National Natural Science Foundation of China under the grants 41174111, 41274121

\section{Reference}

[1] Wright, D. A., Ziolkowski, A., and Hobbs, B. A., 2002, Hydrocarbon detection and monitoring with a multichannel transient electromagnetic (MTEM) survey. The Leading Edge,21, 852-864.

[2] He, J. S., 2010, Full domain electromagnetic method and Pseudorandom signal electric method, China Higher Education Press: Beijing

[3] Tang, J. T., Luo, W. B., 2008, Pseudo random electromagnetic exporation based on invert repeated m-Sequence correlation identification. Chinese J . Geophys. (in Chinese), 51, no. 4, 1226-1233.

[4] Xue. G. Q., Yan, S., Di, Q. Y., Li, X., Wang, R., Li, H., Zhong, H. S. Technical Analysis of Multi-transient Electromagnetic Method. Journal of Earth Sciences and Environment, 2015, 37, no.1, 95-100.

[5] Qi, Y. F., Yin, C. C., Wang, R., Cai, J., 2015, Multi-transient EM fulltime forward modeling and inversion of m-sequences. Chinese $\mathrm{J}$. Geophys. (in Chinese), 58, no.7, 2566-2577.

[6] Wu, X., Xue, G. Q., Di, Q. Y., Zhang, Y. M., Fang, G. Y., 2015 Accurate identification for the electromagnetic impulse response of the earth with psedo random coded wave forms. Chinese J. Geophys. (in Chinese), 58, no.8 2792-2802.

[7] Cheng, P. Q., 2007, Digital signal processor tutorial ( the third edition), Tsinghua university press: Beijing 